\title{
Putting Planetary Materials into the Gap: Insights into Planetary and Stellar Processes from in Situ Measurements in the TEM
}

\author{
Thomas Zega \\ University of Arizona, Tucson, Arizona, United States
}

The chemical and physical origins of our solar system are stored within the solid materials leftover from its birth. Such planetary materials include meteorites, interplanetary dust particles, and samples from other bodies such as the Moon, asteroids, and comets. Interrogation of the crystal chemistry and structure of the minerals within these samples can therefore provide us with insight into the thermodynamic conditions, timescales, and astrophysical settings of their origins.

Studies focused on understanding the origins of planetary materials are generally static. In other words, nature performed the experiment some $\geq 4.567$ billion years ago, and so we reverse engineer the chemistry and physics of this early time through characterization of the solids leftover from solar-system formation. Planetary materials formed or were subsequently altered through a variety of processes including, e.g., condensation from a vapor, gas-solid reactions, melt solidification, liquid-solid reactions, impacts, and ion bombardment. Such processes occurred in environments that are generally extreme (low pressure and high temperature) relative to what we encounter in the laboratory under STP. Thus, novel in situ techniques in the transmission electron microscope (TEM) offer a new means of accessing such conditions and investigating chemical and physical processes on planetary and synthetic analog materials in real time. Below I provide two examples of the way in which our planetary-materials research group has been using in situ TEM techniques to explore planetary and stellar processes.

Planetary bodies that lack atmospheres are subject to micrometeoroid impacts and irradiation from the solar wind. Referred to as space weathering, such processing alters the microstructural and chemical properties of soils on airless bodies like the Moon [1]. The products of space weathering include, e.g., breakdown of long-range atomic order on the grain surface, vesicles within grains, and nanometer-sized Fe-bearing particles [2]. The latter have long been known to contribute significantly to the optical properties of lunar soil and hence the reflectance spectra acquired from them [3]. Understanding how such nanophase $\mathrm{Fe}(\mathrm{npFe})$ forms and how it affects optical properties could therefore help us unravel weathering processes on the Moon, and by analogy, those of other airless bodies, e.g., asteroids. Several hypotheses were proposed for the origin of npFe in lunar soils. These include: (1) vaporization and dissociation of Fe-oxide molecules during micrometeorite impact and preferential condensation and reduction of $\mathrm{Fe}$ as nanoparticles [4]; (2) solar-wind implanted $\mathrm{H}$ acting as a reducing agent in grains during melting processes associated with micrometeorite impacts [1]; and (3) ion-irradiation causing preferential sputtering of $\mathrm{O}$ atoms in surface grains and reduction of the target [1]. Using a recently developed Hitachi heating holder with MEMS-based heating chips, we tested hypothesis (1) inside of the TEM [5]. Briefly, a fine-grained sample of mature lunar soil from the NASA Apollo 17 Mission (79221) was drop cast onto a MEMS chip and thermally shocked to $\sim 940{ }^{\circ} \mathrm{C}$ and back to room temperature in < $1 \mathrm{~s}$ using the Hitachi HF3300 TEM at the University of Toronto. Particles of npFe begin to form at $575{ }^{\circ} \mathrm{C}$ and electron energyloss spectroscopy shows that they are composed of Fe metal. These in situ experiments accurately reproduce the short-duration, high-temperature conditions that lunar soil grains could have experienced during micrometeorite weathering and support hypothesis (1). These results will be discussed at the meeting.

Primitive meteorites contain within them the preserved ashes of ancient stars. These 'presolar' grains condensed in gaseous circumstellar environments and made their way through the interstellar medium to 
our local part of the galaxy where the solar system was forming some 4.56 billion years ago. SiC and graphite are among the largest known types of presolar stardust grains preserved inside of primitive meteorites [6]. SiC was shown to occur predominantly in the 3C polytype likely reflecting the thermodynamic conditions in which it formed [7]. However, some grains of $\mathrm{SiC}$ and other metal-carbides have been observed inside of grains of graphite [8], raising questions regarding the sequence in which these materials condensed from the vapor in their host circumstellar envelope. To better understand the origins of such materials, and moreover, how reduced $\mathrm{C}$ can form in an otherwise H-rich environment [9], we performed in situ heating and ion irradiation experiments with synthetic forms of SiC [10]. Briefly, 3C-SiC (99\% purity) nanoparticles (45 to $65 \mathrm{~nm}$ ) were drop cast onto SiN films as part of the MEMS platform described above for in situ heating in the TEM. The MEMS chips were loaded into the Hitachi heating holder and the Hitachi H9000 TEM at the intermediate voltage electron microscope tandem facility (IVEM) at Argonne National Laboratory. The sample was heated at $5{ }^{\circ} \mathrm{C} / \mathrm{min}$ to $1000{ }^{\circ} \mathrm{C}$ under a

vacuum of $10^{-6} \mathrm{~Pa}$ (very close to circumstellar conditions). Once that temperature was reached, we irradiated the sample at $150 \mathrm{keV}$ using Xe ions. The rapid heating and irradiation were performed to mimic shock heating in circumstellar shells. Graphite and nanobuds of $\mathrm{C}$ formed on the surface of the $\mathrm{SiC}$ suggesting that $\mathrm{C}_{60}$ and derivatives can undergo facile formation as a result of the shock heating and ion bombardment. Such heating and bombardment could occur in various stages of stellar evolution which we will discuss at the meeting [11].

\section{References}

[1] B. Hapke,0 Journal of Geophysical Research: Planets 106 (2001), p. 10,039.

[2] L.P. Keller and D. S. McKay, Geochimica et Cosmochimica Acta 61 (1997), p. 2331.

[3] C. Pieters et al., Meteoritics \& Planetary Science 35 (2000), p. 1101.

[4] L.P. Keller et al., Lunar and Planetary Science Conference 29 (1998), abstract \#1762.

[5] M.S. Thompson et al., Meteoritics \& Planetary Science 52 (2017), p. 413.

[6] E. Zinner, Treatise on Geochemistry vol. 1 (2015), p. 181.

[7] T. Daulton et al., T Daulton et al., Science 296, (2002), p. 1852.

[8] K. Croat et al., The Astronomical Journal 139, (2010), p. 2159.

[9] Y. Zhang and S., Kwok The Astrophysical Journal 730 (2011), p.1.

[10] J. Bernal et al., The Astrophysical Journal Letters 883 (2019), p. 1.

[11] I thank Sachiko Amari, Jacob Bernal, Pierre Haenecour, Jane Howe, Michelle Thompson, and Lucy Ziurys for their collaboration. I thank Doug Perovic and Hitachi High Technologies for access to the HF3300 at the University of Toronto. Research supported by NASA grants NNX15AJ22G and 80NSSC19K0509. I acknowledge NASA (grants \#NNX12AL47G and \#NNX15AJ22G) and NSF (grant \#1531243) for funding of the instrumentation in the Kuiper Materials Imaging and Characterization Facility at the Lunar and Planetary Laboratory, University of Arizona. 